\title{
Photometric Observations of Recent Supernovae
}

\author{
D.Yu. Tsvetkov
}

Sternberg Astronomical Institute, Universitetski pr.13, 119992, Moscow, Russia;

tsvetkov@sai.msu.su

Summary. Regular photometric observations of sufficiently bright northern supernovae are carried out at Sternberg Astronomical Institute's observatories. Since 1998 the observations of more than 60 supernovae were obtained on about 150 nights with different telescopes and detectors. We present the data of the observation program, the parameters of light curves for $18 \mathrm{SNe}$ and the light curves for SNe 1999aa, 2001B, 2002bo.

\section{Introduction}

The light curves are among the major sources of information about the nature of supernovae. They are the main tool for the use of SNe as distance indicators, and can also be used for SN classification and for constraining theoretical models. The observational data have been substantially increased during last years. The modern observational programs contributed high-quality multicolor CCD photometry with good temporal sampling for many SNe. But the rate of SN discovery is so high, that for some sufficiently bright SNe only few photometric data points have been obtained. Often only the low-quality results of amateur groups are available. So, regular photometry of bright $\mathrm{SNe}$ remains an urgent observational task.

SN photometry has been carried out at Sternberg Astronomical Institute since the 1960s, and the most prominent results were obtained for SNe 1981B, $1984 \mathrm{E}, 1984 \mathrm{~L}$, and 1985F [7, 11, 12, 13]. Their light curves were derived from photographic and photoelectric observations. During the 1990 s we had serious difficulties in accomplishing observations. Only since 1998 it was possible to revive the program, using $\mathrm{CCD}$ detectors.

\section{Observations and Reductions}

The observations were carried out with 125-, 60-, 50-, and 38-cm telescopes of Sternberg Institute Crimean observatory and also at 70- and 30-cm telescopes in Moscow. We used CCD cameras AP-7p, ST-8, ST-7 and ST-6 with different sets of filters, and occasionally obtained also photographic and photoelectric observations. The reductions were done in the usual manner. The 
Table 1. Basic data for observation program

\begin{tabular}{lrrrlrrrrrrrr}
\hline SN & $\mathrm{T}_{1}$ & $\mathrm{~T}_{n}$ & $\mathrm{~N}$ & $\mathrm{SN}$ & $\mathrm{T}_{1}$ & $\mathrm{~T}_{n}$ & $\mathrm{~N}$ & $\mathrm{SN}$ & $\mathrm{T}_{1}$ & $\mathrm{~T}_{n}$ & $\mathrm{~N}$ \\
\hline $1997 \mathrm{cx}$ & 19 & 214 & 11 & $1999 \mathrm{em}$ & 5 & 379 & 24 & $2001 \mathrm{dn}$ & 2 & 82 & 17 \\
$1997 \mathrm{dn}$ & 7 & 117 & 8 & $1999 \mathrm{gi}$ & 4 & 354 & 21 & $2001 \mathrm{dp}$ & 4 & 93 & 6 \\
$1997 \mathrm{do}$ & 3 & 118 & 12 & $1999 \mathrm{gn}$ & 103 & 29 & 5 & $2001 \mathrm{ed}$ & 16 & 53 & 6 \\
$1997 \mathrm{dq}$ & 5 & 116 & 10 & $1999 \mathrm{gq}$ & 93 & 15 & 3 & $2001 \mathrm{ef}$ & 11 & 52 & 4 \\
$1997 \mathrm{ef}$ & 3 & 122 & 8 & $2000 \mathrm{C}$ & 45 & 32 & 9 & $2001 \mathrm{en}$ & 41 & 47 & 6 \\
$1997 \mathrm{eg}$ & 79 & 8 & 5 & $2000 \mathrm{E}$ & 13 & 79 & 13 & $2001 \mathrm{fa}$ & 19 & 47 & 6 \\
$1997 \mathrm{ei}$ & 62 & 6 & 4 & $2000 \mathrm{cx}$ & 21 & 103 & 19 & $2001 \mathrm{gd}$ & 94 & 41 & 6 \\
$1998 \mathrm{D}$ & 21 & 11 & 7 & $2000 \mathrm{db}$ & 19 & 100 & 5 & $2002 \mathrm{an}$ & 52 & 51 & 8 \\
$1998 \mathrm{~S}$ & 29 & 366 & 15 & $2000 \mathrm{dk}$ & 3 & 73 & 17 & $2002 \mathrm{ap}$ & 3 & 307 & 23 \\
$1998 \mathrm{aq}$ & 127 & 97 & 6 & $2000 \mathrm{dx}$ & 13 & 67 & 12 & $2002 \mathrm{bo}$ & 6 & 72 & 17 \\
$1998 \mathrm{dh}$ & 31 & 95 & 5 & $2000 \mathrm{ev}$ & 2 & 80 & 6 & $2002 \mathrm{bu}$ & 3 & 57 & 10 \\
$1998 \mathrm{ef}$ & 29 & 30 & 4 & $2000 \mathrm{ew}$ & 1 & 156 & 7 & $2002 \mathrm{cr}$ & 6 & 19 & 4 \\
$1998 \mathrm{es}$ & 2 & 94 & 8 & $2001 \mathrm{~B}$ & 19 & 104 & 14 & $2002 \mathrm{cs}$ & 2 & 19 & 4 \\
$1999 \mathrm{D}$ & 4 & 96 & 10 & $2001 \mathrm{C}$ & 18 & 98 & 8 & $2002 \mathrm{es}$ & 11 & 76 & 5 \\
$1999 \mathrm{X}$ & 20 & 49 & 13 & $2001 \mathrm{G}$ & 16 & 71 & 5 & $2002 \mathrm{hh}$ & 13 & 241 & 19 \\
$1999 \mathrm{aa}$ & 2 & 72 & 17 & $2001 \mathrm{~V}$ & 9 & 84 & 14 & $2002 \mathrm{ho}$ & 14 & 16 & 6 \\
$1999 \mathrm{ac}$ & 16 & 43 & 6 & $2001 \mathrm{X}$ & 17 & 76 & 11 & $2002 \mathrm{hw}$ & 9 & 17 & 11 \\
$1999 \mathrm{an}$ & 7 & 24 & 4 & $2001 \mathrm{ai}$ & 6 & 33 & 3 & $2002 \mathrm{ji}$ & 4 & 112 & 8 \\
$1999 \mathrm{dk}$ & 7 & 82 & 4 & $2001 \mathrm{ay}$ & 8 & 38 & 6 & $2003 \mathrm{Z}$ & 29 & 57 & 4 \\
$1999 \mathrm{dh}$ & 15 & 95 & 4 & $2001 \mathrm{bf}$ & 3 & 133 & 10 & $2003 \mathrm{as}$ & 9 & 72 & 8 \\
$1999 \mathrm{ej}$ & 19 & 41 & 5 & $2001 \mathrm{bg}$ & 2 & 13 & 3 & $2003 \mathrm{cg}$ & 2 & 53 & 8 \\
$1999 \mathrm{el}$ & 12 & 52 & 10 & & & & & & & & \\
\hline
\end{tabular}

magnitude differences between SNe and 1-2 comparison stars were derived by aperture photometry. When the galaxy background near SN was bright and non-uniform, we subtracted the image of the galaxy obtained after fading of SN from the image with SN. On photometric nights we calibrated the comparison stars and determined the transformation equations from instrumental system to the standard one by observing Landolt's [3] and Mermilliod's [6] standard stars, and also clusters M67 [1] and NGC7790 [9].

The brief review of the program is presented in Table 1, where for all SNe with more than 2 observations we report the following data: $T_{1}$ is the time in days from discovery until first observation; $\mathrm{T}_{n}$ is the time from first observation until last observation; $\mathrm{N}$ is the number of nights on which observations were obtained.

\section{Results of Observations}

Observations of $23 \mathrm{SNe}$ have been already reduced. For $5 \mathrm{SNe}$ the data were insufficient to determine the shape of the light curves. The main light curve parameters for $18 \mathrm{SNe}$ are reported in Table 2, where $m$ is magnitude at 
Table 2. Parameters of light curves for SNe

\begin{tabular}{llllllll}
\hline SN & SN Type & $m$ & $\Delta m_{15}$ & $\mu$ & $A_{B}$ & $E(B-V)$ & $M$ \\
\hline $1997 \mathrm{cx}$ & IIL & $14.2 R$ & & 32.0 & 0.10 & & $-17.9 R$ \\
$1997 \mathrm{do}$ & Ia & $14.2 B$ & 1.3 & 33.3 & 0.27 & & $-19.4 B$ \\
$1997 \mathrm{ef}$ & IcPec & 16.5 & & 33.3 & 0.18 & & -17.1 \\
$1998 \mathrm{D}$ & Ia & $15.5 R$ & 1.3 & 33.7 & 0.06 & 0.15 & $-18.6 R$ \\
$1998 \mathrm{dh}$ & Ia & 13.9 & 1.0 & 32.6 & 0.29 & 0.1 & -19.0 \\
$1998 \mathrm{ef}$ & Ia & $15.0 R$ & 1.1 & 34.4 & 0.32 & 0.15 & $-19.7 R$ \\
$1998 \mathrm{es}$ & IaPec & 13.8 & 0.9 & 33.1 & 0.14 & 0.16 & -19.8 \\
$1999 \mathrm{D}$ & IIP & 17.4 & & 33.4 & 0.07 & & -16.1 \\
$1999 \mathrm{X}$ & Ia & 16.3 & 1.0 & 35.0 & 0.14 & & -18.8 \\
$1999 \mathrm{aa}$ & IaPec & 14.8 & 1.0 & 33.9 & 0.17 & & -19.2 \\
$1999 \mathrm{gi}$ & IIP & 14.9 & & 30.3 & 0.07 & 0.21 & -16.1 \\
$1999 \mathrm{el}$ & IIL & 14.9 & & 31.9 & 1.58 & & -18.2 \\
$2000 \mathrm{E}$ & Ia & 13.7 & 1.3 & 31.9 & 1.58 & 0.36 & -19.3 \\
$2001 \mathrm{~B}$ & Ib & 15.0 & & 32.0 & 0.54 & & -17.5 \\
$2001 \mathrm{~V}$ & Ia & 14.5 & 0.9 & 34.1 & 0.08 & & -19.5 \\
$2001 \mathrm{X}$ & IIP & 15.3 & & 32.0 & 0.17 & & -16.8 \\
$2002 \mathrm{ap}$ & IcPec & 12.4 & & 29.5 & 0.31 & 0.09 & -17.4 \\
$2002 \mathrm{bo}$ & Ia & 13.6 & 1.1 & 31.8 & 0.11 & 0.35 & -19.2 \\
\hline
\end{tabular}

plateau for SNe IIP and at maximum light for SNe of other types, in the $V$ band unless followed by symbol denoting other band; $\Delta \mathrm{m}_{15}$ is only a rough estimate, based on comparison with template SN Ia light curves; distance modulus $\mu$ is taken from [14] or estimated from radial velocity, corrected for Virgocentric infall, with $H_{0}=75 \mathrm{~km} \mathrm{~s}^{-1} \mathrm{Mpc}^{-1}$; galactic absorption $A_{B}$ is from [8], and $E(B-V)$ is estimated by comparing the color curves of $\mathrm{SNe}$ under study with those for which extinction is known to be negligible, for SNe 1999gi and 2002ap it is taken from [4,5]; the absolute magnitude $M$, also in the $V$ band unless another band is noted, is derived using the reported apparent magnitude, distance and extinction, for SNe with no data on $E(B-$ $V$ ) we assumed negligible reddening in parent galaxies and accounted only for galactic extinction.

The main conclusion is that all studied SNe are quite similar to the typical objects of their classes, as regarding shape of the light curves and absolute magnitudes. Among two peculiar SNe Ia (similar to SN 1991T) SN 1998es was slightly overluminous, while absolute magnitude of SN 1999aa was quite close to the average value for SNe Ia. The light curves for SNe 1999aa, 2001B and 2002bo are presented in Figs. 1-3. 


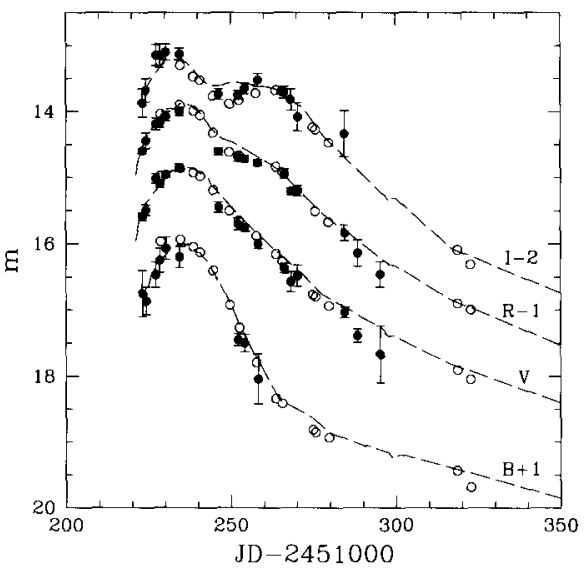

Fig. 1. Light curves of SN 1999 aa in the $B, V, R, I$ bands. Filled symbols show our results, open symbols are the data of [2]. The dashed lines are the light curves of SN IaPec $1991 \mathrm{~T}$.

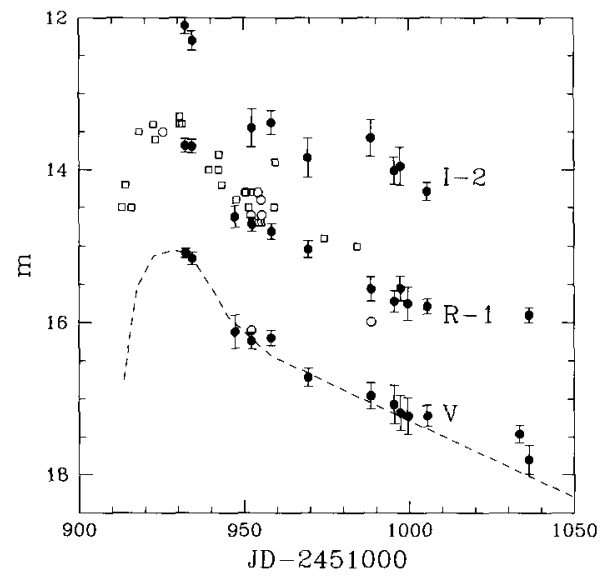

Fig. 2. Light curves of $\mathrm{SN} 2001 \mathrm{~B}$ in the $V, R, I$ bands. Filled symbols show our data, open symbols are the data from IAU Circulars and VSNET. The dashed line is $V$ light curve of $\mathrm{SN} \mathrm{Ib} 1984 \mathrm{~N}$.

\section{References}

1. C. Chevalier, S.A. Ilovaisky: Astrophys. J. Suppl. 90, 225 (1991)

2. K. Krisciunas, A. Diercks, N.C. Hastings et al: Astrophys. J. 539, 658 (2000)

3. A. Landolt: Astron. J. 97, 337 (1992)

4. D.C. Leonard et al. : Astron. J. 124, 2490 (2002)

5. P.A. Mazzali et al. : Astrophys. J. 572, 61 (2002) 


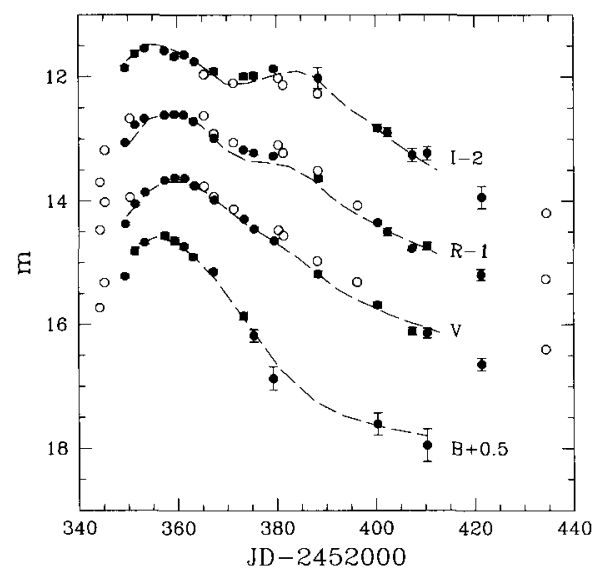

Fig. 3. Light curves of SN 2002 bo in the $B, V, R, I$ bands. Filled symbols represent our data, open symbols show the data of [10]. The dashed lines are the light curves of SN Ia 1998bu.

6. J.-C. Mermilliod: "The General Catalogue of Photometric Data" obswww. unige.ch/gcpd

7. N.V. Metlova, V.G. Metlov, D.Yu. Tsvetkov: Inform. Bull. Var. Stars 2780, 1 (1985)

8. D.J. Schlegel, D.P. Finkbeiner, M. Davis: Astrophys. J. 500, 525 (1998)

9. P.B. Stetson: Pub. Astron. Soc. Pacific 112, 925 (2000)

10. G.M. Szabo et al. : astro-ph 0306606 (2003)

11. D.Yu. Tsvetkov: Astron. Zhurnal 8, 219 (1982)

12. D.Yu. Tsvetkov: Astron. Zhurnal 12, 784 (1986)

13. D.Yu. Tsvetkov: Astron. Zhurnal 13, 894 (1987)

14. R.B. Tully: Nearby Galaxies Catalog (Cambridge University Press, Cambridge 1988) 